\title{
Comparative study of Apparent Diffusion Coefficient Values and Ultrasonography in Evaluation of Adult Chronic Kidney Disease Patients
}

\author{
Vijaypavan Kumar Dasarjư ${ }^{1}$, Sree Hari P², Anusha N ${ }^{3}$, Himaja $\mathbf{G}^{4}$, Prashanthi ${ }^{5}$, Sudhakar $\mathbf{V}^{6}$ \\ ${ }^{1}$ Assistant Professor, Department of Radiodiagnosis, Gandhi Medical College Secunderabad Telangana, ${ }^{2}$ Professor \& \\ HOD, Department of Radiodiagnosis, Gandhi Medical College Secunderabad Telangana, ${ }^{3} J u n i o r$ Resident, Department of \\ Radiodiagnosis, Gandhi Medical College, Secunderabad, Telangana, 'Junior Resident, Department Of Radiodiagnosis, Gandhi \\ Medical College, Secunderabad, 5Junior Resident, Department of Radiodiagnosis, Gandhi Medical College, Secunderabad, \\ ${ }^{4}$ Mri Technician, Department of Radiodiagnosis, Gandhi Medical College, Secunderabad, India
}

Corresponding author:. Dr. Sree Hari P, Professor \& HOD, Department of Radiodiagnosis, Gandhi Medical College, Secunderabad, Telangana, India

DOI: http://dx.doi.org/10.21276/ijcmsr.2020.5.1.43

BY-NC-ND

How to cite this article: Vijaypavan Kumar Dasarju, Sree Hari P, Anusha N, Himaja G, Prashanthi K, Sudhakar V. Comparative study of apparent diffusion coefficient values and ultrasonography in evaluation of adult chronic kidney disease patients. International Journal of Contemporary Medicine Surgery and Radiology. 2020;5(1):A197A202.

\section{A B S T R A C T}

Introduction: Diffusion-weighted magnetic resonance imaging (DWI) is a non-invasive method sensitive to water motion in the tissue.Diffusion weighted magnetic resonance imaging (DW-MRI) in evaluation of renal diseases is an evolving field and its utility is to be understood and it has the potential to become an effective imaging biomarker. Study objective was to evaluate the role of diffusion weighted MRI in staging chronic kidney disease by apparent diffusion coefficient values and correlating ADC values with CKD staging and Ultrasound grading.

Material and Methods: A prospective study done in adult patients with chronic kidney disease from January 2019 to august 2019. A total of 50 cases were included in the study. Serum markers of renal failure were noted, CKD staging was done by calculating eGFR using MDRD formula followed by Ultrasound evaluation for parenchymal Grading.Later, MRI was done and $A D C$ values of parenchyma were determined. CKD staging and ultrasound grading of parenchyma was correlated with ADC values of parenchyma

Results: In our study,out of total 50 cases, majority number of cases were seen in male population(64\%), with younger agegroup (21 - 30 years) predominance-16 cases (32\%). Clinically based on eGFR calculation, CKD stage 1 cases were4(8\%),Stage II - 2(4\%), Stage III - 8(16\%), Stage IV-14(28\%) and stage V cases 22(44\%). On ultrasound grading, Grade I cases-10(20\%), Grade II cases-16(32\%), Grade III cases - 14(28\%) and Grade IV cases-10(20\%). ADC values of stage IV and V were correlating with CKD staging and Ultrasound Grading (90 to 100\%) whereas clinically CKD stage I and Stage II, and USG grade I and II cases ADC values are not correlating as most of cases were showing Stage III ADC values. Linear correlation was seen with $A D C$ values and e GFR and inverse correlation was seen with serum markers.

Conclusion: ADC values of renal parenchyma is a useful tool in evaluating renal dysfunction, which will make MR imaging of kidney as one step modality for renal evaluation

Keywords: Apparent Diffusion Coefficient, Chronic Kidney Disease,Ultrasound, e GFR.

\section{INTRODUCTION}

Chronic kidney disease is recognised as world wide leading health problem.Serum markers such as creatinine and blood urea nitrogen level and estimated glomerular filtration rate (eGFR) are useful parameters for estimating renal function, however they depend on age and body mass index of patient and cannot be used to evaluate single kidney function. ${ }^{1,2}$ Hence because of these limitations, imaging techniques such as ultrasonography, diffusion weighted magnetic resonance imaging (DW-MRI) are gaining importance in evaluation of renal function, Though ultrasonography is preferred modality, the lack of specificity in assessing renal disease emphasises on using better imaging modality, The apparent diffusion coefficient is a quantitative parameter calculated from DWMRI images and represents water diffusion in extracellular and extra vascular space and capillary perfusion,Diffusion weighted imaging(DWI) in renal diseases is an evolving field and many investigators used it to characterize renal parenchymal disease. . $^{3,4,5,6,7,8}$

The purpose of this study is to determine the apparent diffusion coefficient (ADC) values of renal parenchyma and its relation with ultrasonographic grading of $\mathrm{CKD}$, serum 
markers of renal function and staging of chronic kidney disease.

\section{MATERIAL AND METHODS}

This was the prospective single institution study approved by institute ethical committee and was done after informed consent was taken from patients. Our study was conducted fromJanuary 2019 to august 2019. The total number patients included were 50 , with male predominance $(64 \%$ male $36 \%$ female)with a mean age group of 42 years. For eGFR calculation, patients demographic data and serum investigations were collected from records.

\section{CKD grading}

eGFR was calculated from MDRD formula ${ }^{9}$ using body surface area, age, sex, race, serum creatinine level Formula $\mathrm{e}$ GFR $\mathrm{ml} / \mathrm{min} / 1.73 \mathrm{~m} 2=194 \mathrm{x}$ creatinine $-1.094 \mathrm{x}$ age 0.287 (0.739 if female). Patients were classified into 5 stages with e GFR value.

Stage 1: eGFR; $\geq 90 \mathrm{~mL} / \mathrm{min} / 1.73 \mathrm{~m} 2$ (kidney damage with normal or increased eGFR)

Stage 2: eGFR; $60-89 \mathrm{~mL} / \mathrm{min} / 1.73 \mathrm{~m} 2$ (kidney damage with a mild reduction in eGFR).

Stage 3: eGFR; $30-59 \mathrm{~mL} / \mathrm{min} / 1.73 \mathrm{~m}$ (moderate reduction in eGFR).

Stage 4: eGFR; $15-29 \mathrm{~mL} / \mathrm{min} / 1.73 \mathrm{~m} 2$ (severe reduction in eGFR).

Stage 5: eGFR; < $15 \mathrm{~mL} / \mathrm{min} / 1.73 \mathrm{~m} 2$ (kidney failure).

\section{Ultrasonography}

Ultrasound was done on GE voluson P5 and renal parenchymal echogenicity was evaluated. Grading of renal parenchymal echogenicity was done. ${ }^{10}$

Grade 1-Normal sized kidney, cortical echogenicity is same as liver, with well maintainedcortico-medullary differentiation.

Grade 2- Normal sized kidney, cortical echogenicity is greater than that ofliver, with maintained cortico-medullary differentiation.

Grade 3 - Normal sized kidney, cortical echogenicity is more than that of liver, decreased cortico-medullary differentiation.

Grade 4- Reduced renal length, cortical echogenicity is more than that of liver, with poorly maintained cortico-medullary differentiation.

\section{MRI}

All the patients underwent MRI on $1.5 \mathrm{~T}$ (seimensavanto Germany) using phased array body coil. Image protocols included were T1 axial, T2 axial, coronal, sagittal planes. DWI was done at b values $0,500,1000 \mathrm{~s} / \mathrm{mm}$. ADC maps derived automatically on voxel by voxel basisThe ADC values are expressed as mean + standard deviation as A X $10^{-3} \mathrm{~mm}^{2} / \mathrm{s}$ upto 4 decimal places. ${ }^{11}$ Region of interest (ROI) for quantitative measurement of ADC were placed on renal parenchyma with out preference for cortex /medulla., ${ }^{8,12,13}$ Values for each kidney were recorded and relationship between $\mathrm{ADC}$ values and stage of $\mathrm{CKD}$, ultrasonography grade of renal parenchyma were evaluated for each patient.

\section{RESULT}

In our study, out of total 50 cases, majority number of cases were seen in male population-32 cases (64\%), with younger age group (21 - 30 years) predominance-16 cases (32\%). Clinically based on eGFR calculation, CKD stage 1 cases were4(8\%), Stage II - 2(4\%), Stage III - 8(16\%), Stage IV$14(28 \%)$ and stage $V$ cases 22(44\%). On ultrasound grading, Grade I cases- 10(20\%), Grade II cases-16(32\%), Grade III cases - 14(28\%) and Grade IV cases-10(20\%). ADC values of stage IV and $\mathrm{V}$ were correlating with CKD staging and Ultrasound Grading (90 to 100\%) whereas clinically CKD stage I and Stage II, and USG grade I and II cases ADC values are not correlating as most of cases were showing Stage III ADC values. Linear correlation was seen with

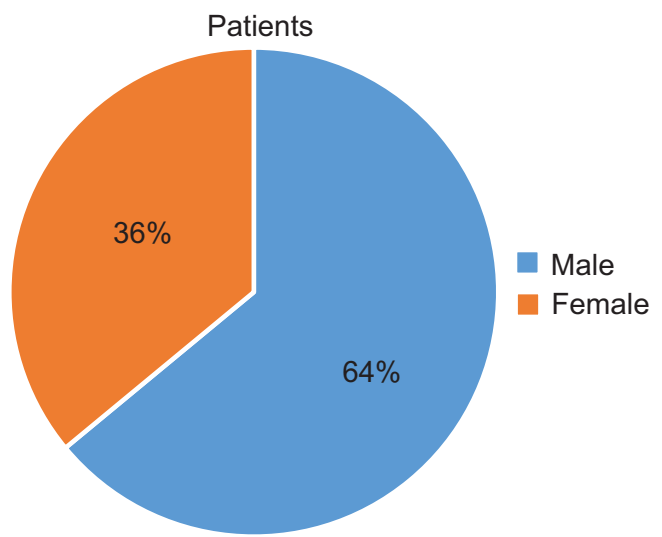

Graph-1: Pie diagram showing gender wise distribution:

Bar graph depicting age wise distribution

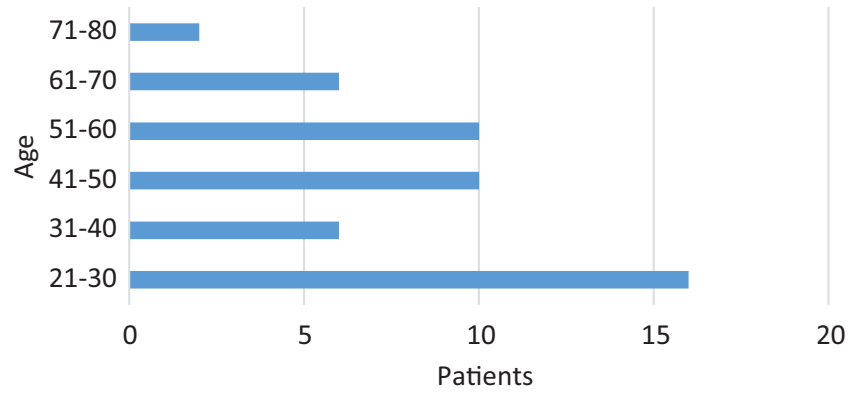

Graph-2: Bar graph depicting age wise distribution



Graph-3: Pie diagram showing ultrasonography grading: (\% of patients) 


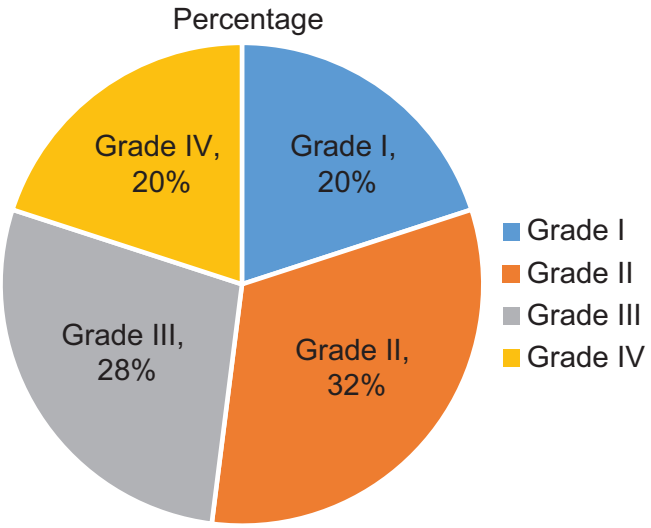

Graph-4: Pie diagram showing eGFR CKD grading: (\% of patients)

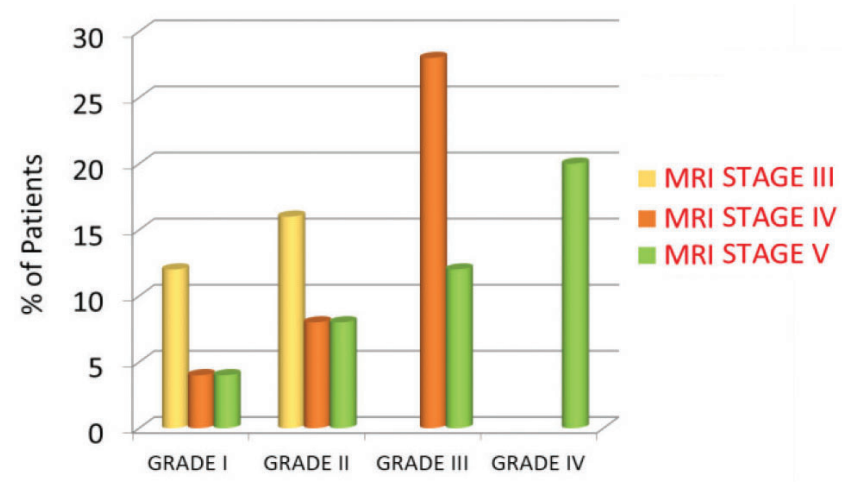

\section{Graph-5:}
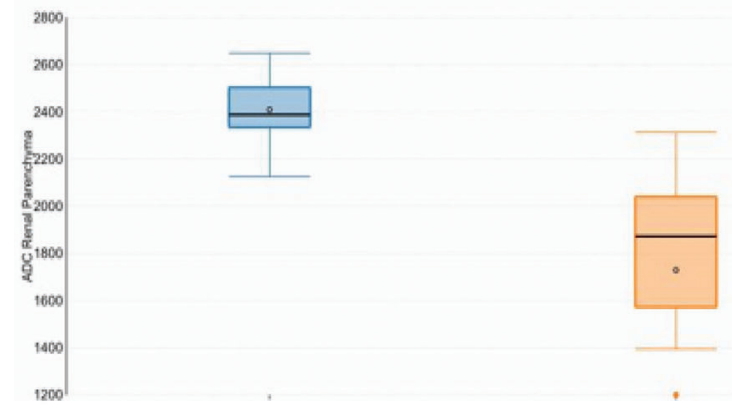

Normal Renal Function.

Renal Dysfunction

Graph-6: ADC values and renal function

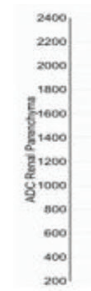



Stage 3



Stage 4

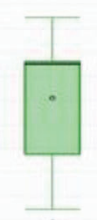

Stage 5
Graph-7: ADC values and stages of CKD

ADC values and e GFR and inverse correlation was seen with serum markers

Out of total 50 cases, majority number of cases were seen in male population-32 cases (64\%) (fig-1)

Majority of cases were seen in younger age group population

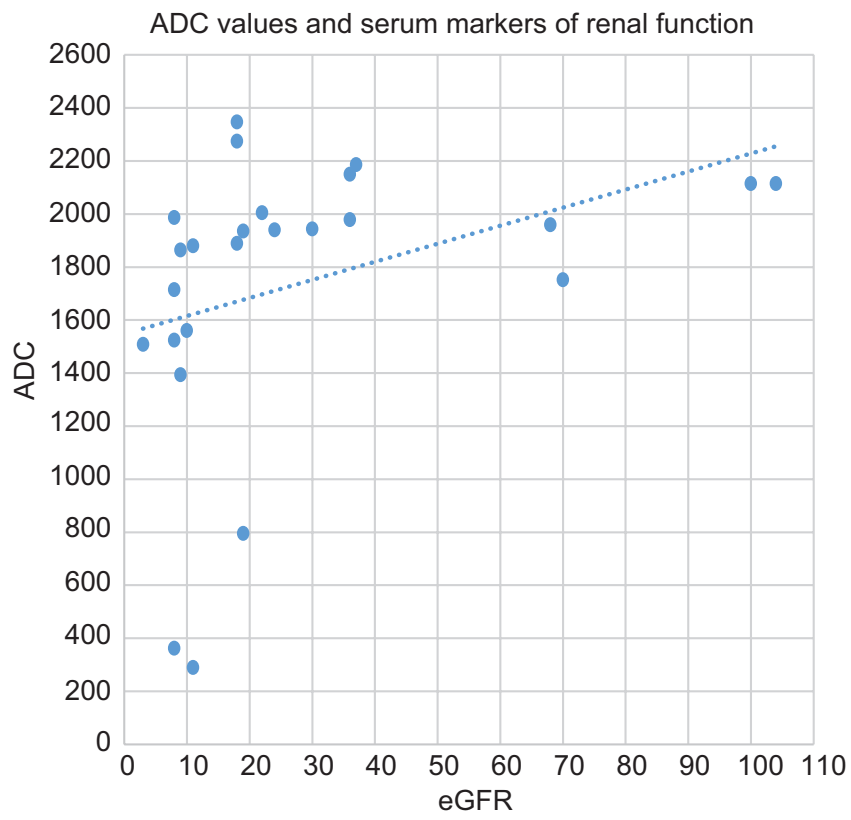

Graph-8: ADC values and serum markers of renal function

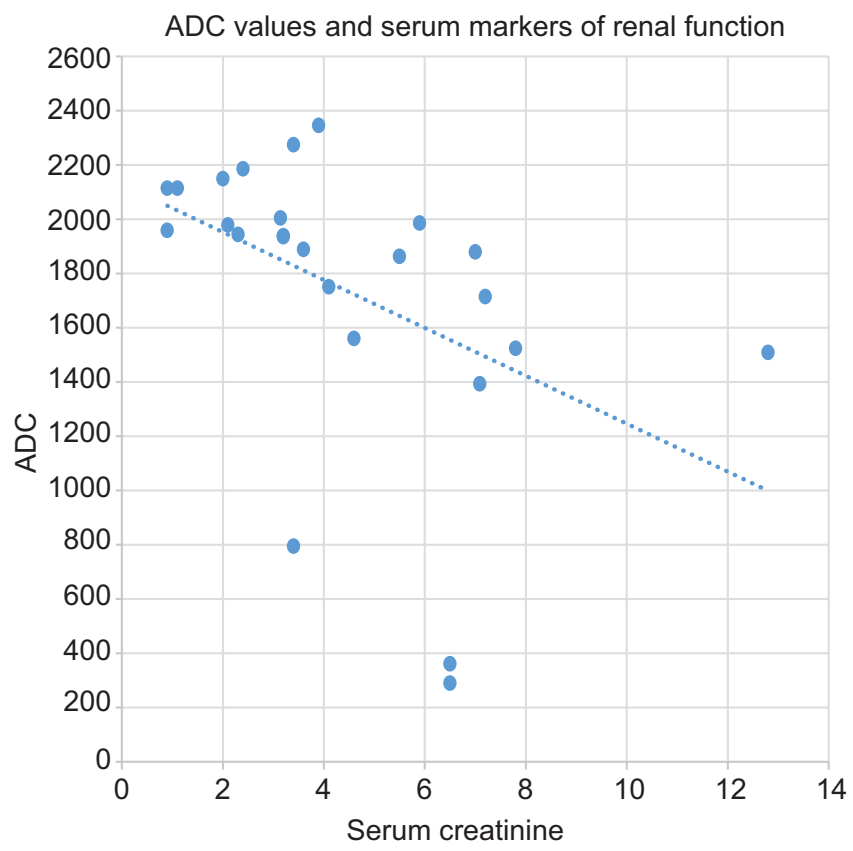

Graph-9: ADC values and serum markers of renal function

that is between 21-30years of age constituting 32\% (16 out of 50 cases) (fig-2).

On ultrasound grading, Grade I cases- 10 (20\%), Grade II cases-16 (32\%), Grade III cases - 14(28\%) and Grade IV cases-10 (20\%) (fig-3).

Clinically based on eGFR calculation, CKD stage 1 cases were4(8\%), Stage II - 2(4\%), Stage III - 8(16\%), Stage IV$14(28 \%)$ and stage $V$ cases $22(44 \%)$ (fig-4).

The adc values $\left(\mathrm{x} 10^{-3} \mathrm{~mm}^{2} / \mathrm{s}\right)$ of stage 3 were in the range of $2.170-2.410$, stage $4: 1.630-2.050$ and stage $5: 1.340$ 1.700 which were taken as reference from previous studies (table-1).

$\mathrm{ADC}$ values of stage IV and $\mathrm{V}$ were correlating with $\mathrm{CKD}$ staging and Ultrasound Grading (90 to 100\%) whereas clinically CKD stage I and Stage II, and USG grade I and 


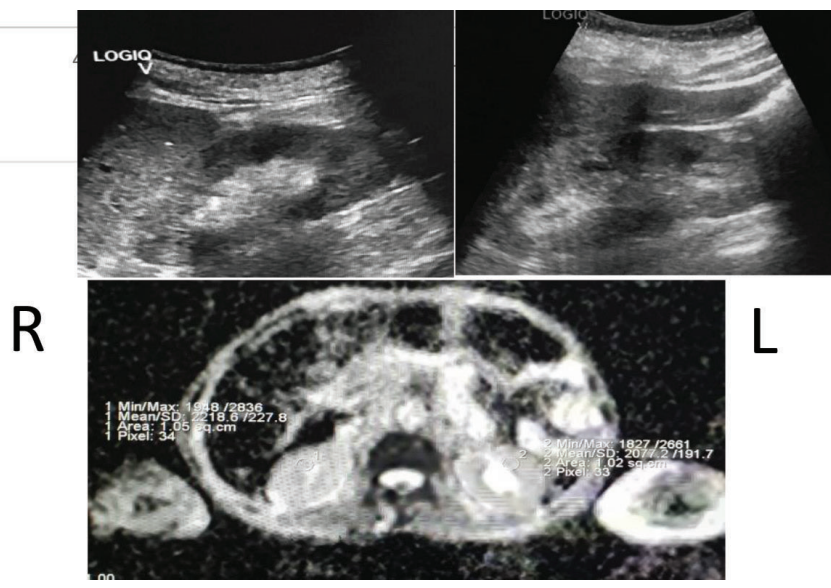

Figure-1: A 65year old male presented with decreased urine output and bilateral loin pain

Serum creatinine: $2.1 \mathrm{mg} / \mathrm{dl}$

eGFR: $36 \mathrm{ml} / \mathrm{min} / 1.73 \mathrm{~m} 2-\mathrm{CKD}$ Stage III

USG: Grade III

MRI: Mean ADC values of 2218/2027- MRI ADC stage III
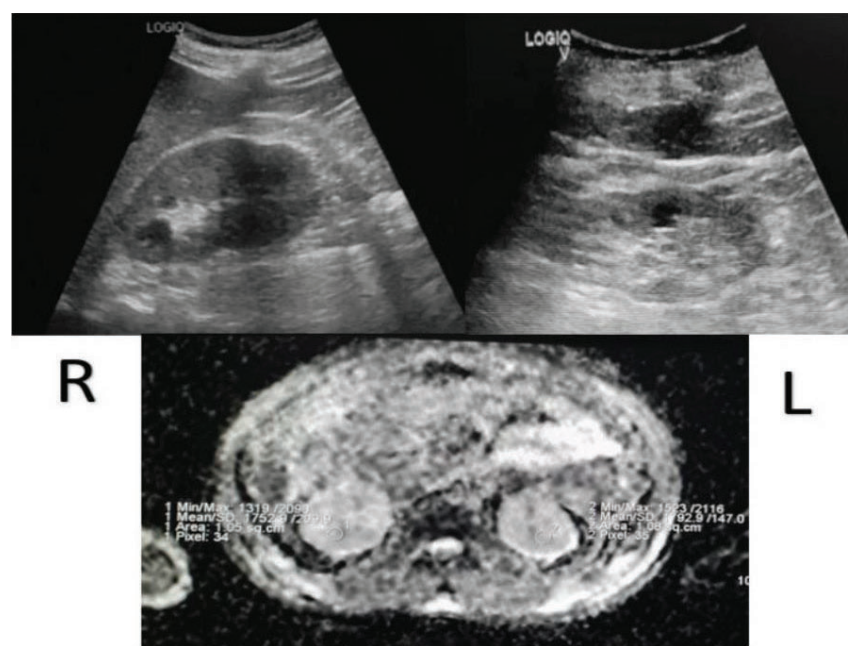

Figure-2: A 32 year old male presented with decreased urine output with giddiness, $\mathrm{k} / \mathrm{c} / \mathrm{o}$ young hypertension

Serum creatinine: $4.1 \mathrm{mg} / \mathrm{dl}$

eGFR: $17 \mathrm{ml} / \mathrm{min} / 1.73 \mathrm{~m} 2$ - CKD grade IV

USG: Grade III

MRI: mean ADC values of 1752/1792-MRI ADC stage IV

II cases ADC values are not correlating as most of cases were showing Stage III ADC values (table-2).

Box-and-whisker plot of renal parenchymal ADC in patients with renal dysfunction and those with normal renal function showing lower ADC values associated with renal dysfunction.

The lines within boxes represent median value. The top and bottom of boxes (hinges) represent the 25 th and 75 th percentiles of the data values. The T-bars that extend from the boxes (whiskers) are expected to include approximately 95\% of the data (assuming normal distribution) (fig-6).

The mean ADC values of renal parenchyma in patients with renal dysfunction was significantly lower than in patients with normal renal function $(1.6051 \pm 0.2851$ vs. $2.4094 \pm$ $0.1246\left(\times 10^{-3} \mathrm{~mm}^{2} / \mathrm{s}\right)$.

Box-and-whisker plot of renal parenchymal ADC in

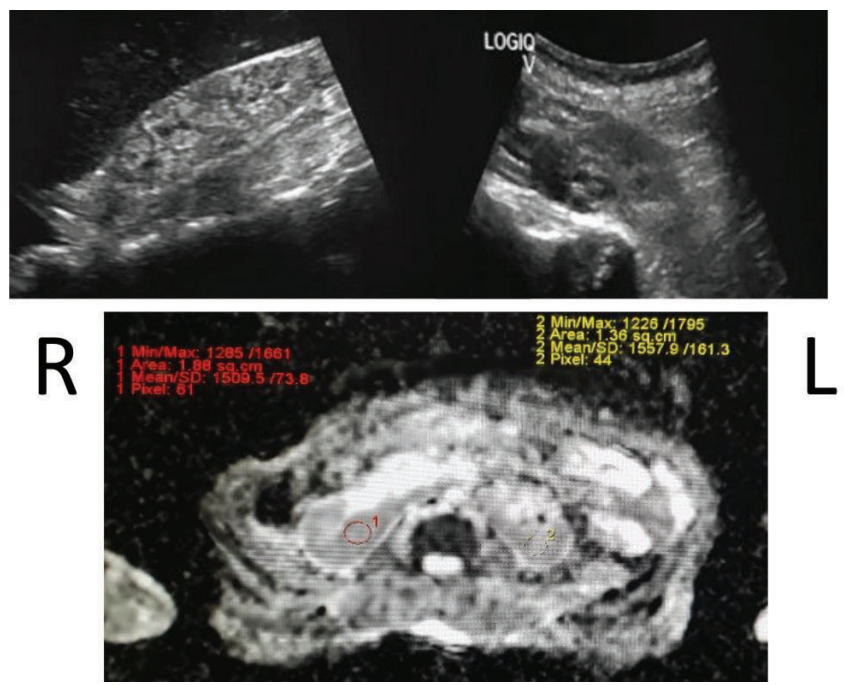

Figure-3: A 45 year old female with generalised edema, decreased urine output, shortness of breath

Serum creatinine: $12.8 \mathrm{mg} / \mathrm{dl}$

eGFR: $3.2 \mathrm{ml} / \mathrm{min} / 1.73 \mathrm{~m} 2-\mathrm{CKD}$ stage $\mathrm{V}$

USG: Grade IV

MRI: Mean ADC values of 1509/1557 -MRI ADC Stage V
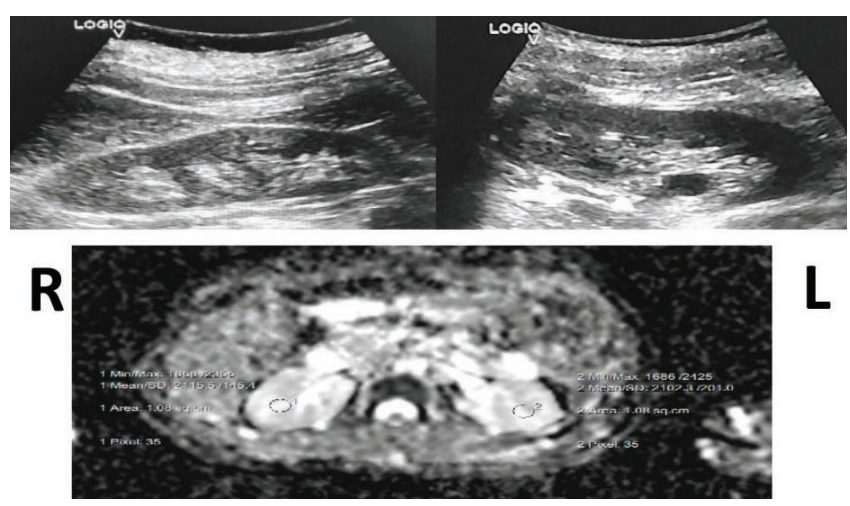

Figure-4: A 40 year old female with loin pain Serum Creatnine: $0.9 \mathrm{mg} / \mathrm{dl}$

eGFR: $90 \mathrm{ml} / \mathrm{min} / 1.73 \mathrm{~m} 2$ - CKD stage I

USG: Grade I

MRI: mean ADC of 2115/2150- - MRI ADC stage III

different stages of $\mathrm{CKD}$ showing decreasing $\mathrm{ADC}$ values with increasing stage of CKD

The mean $\mathrm{ADC}$ values of different stages of $\mathrm{CKD}$ were significantly different from each other and showed decreasing trend with increasing stage $\left(2.01504 \pm 0.1243\left(\times 10^{-3} \mathrm{~mm}^{2}\right.\right.$ /s) for stage $-3,1.8263 \pm 0.2117\left(\times 10^{-3} \mathrm{~mm}^{2} / \mathrm{s}\right)$ for stage-4, and $1.2208 \pm 0.1853\left(\times 10^{-3} \mathrm{~mm}^{2} / \mathrm{s}\right)$ for stage -5$)($ fig- -7$)$.

Within the CKD study group $(n=50)$, a significant linear correlation was found between renal parenchymal ADC values and eGFR (fig-8). There was a significant inverse correlation between ADC values of renal parenchyma and $\mathrm{S}$ Cr levels (Fig- 9).

\section{DISCUSSION}

According to our study, chronic kidney disease is more common in males with mean age of 42 years, which is similar to observations in previous studies.

By grading the renal parenchymal changes, the severity of 


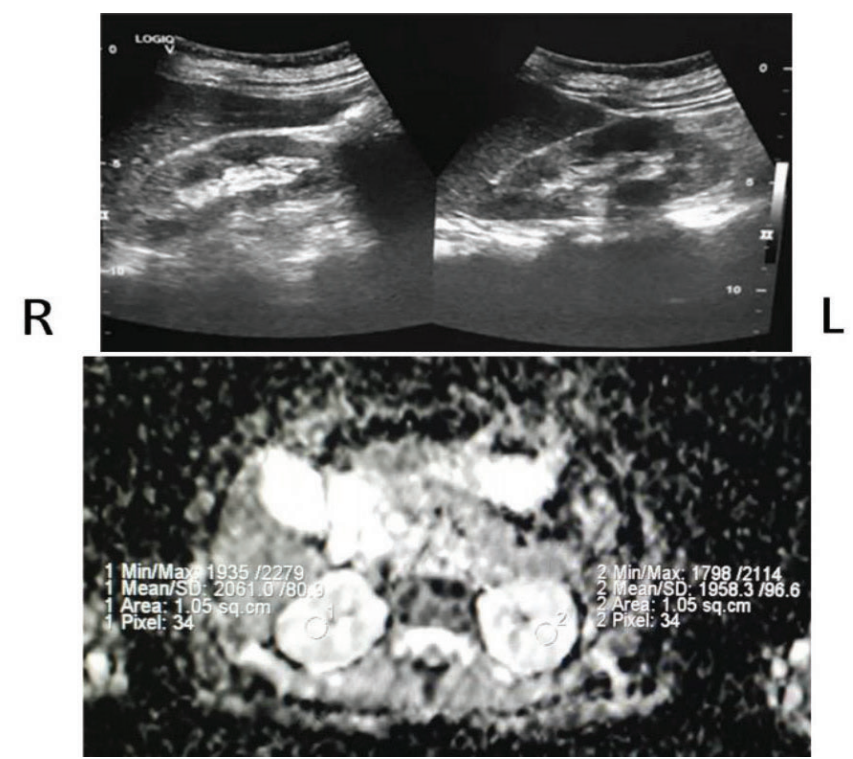

Figue-5: A 44year old male presented with bilateral loin pain. Serum Creatnine: $1.3 \mathrm{mg} / \mathrm{dl}$ eGFR:68ml/min/1.73m2- CKD Stage II

USG: Grade II

MRI: Mean ADC values of 2061/1958 -MRI ADC Stage III

\begin{tabular}{|l|c|c|}
\hline CKD grading & ADC (X 10-6 $\mathbf{~ m m 2 / s )}$ & \% of Patients \\
\hline Stage 3 & $2170-2410$ & 28 \\
\hline Stage 4 & $1630-2050$ & 28 \\
\hline Stage 5 & $1340-1700$ & 44 \\
\hline \multicolumn{2}{|c|}{ Table-1: CKD grading with reference ADC values } \\
\hline
\end{tabular}

\begin{tabular}{|l|l|l|}
\hline $\begin{array}{l}\text { Ultrasound grad- } \\
\text { ing -\% of patients }\end{array}$ & $\begin{array}{l}\text { CKD staging } \\
\text { (e GFR)-\% of } \\
\text { patients }\end{array}$ & $\begin{array}{l}\text { ADC values-\% of } \\
\text { patients }\end{array}$ \\
\hline \multirow{2}{*}{ Grade I- 20 } & CKD stage I -8 & MRI stage III -12 \\
\cline { 2 - 2 } & CKD stage II - 4 & \\
\hline Grade II - 32 & CKD stage III A- - & MRI stage III -16 \\
\hline \multirow{2}{*}{ Grade III - 28 } & CKD stage III B-8 & MRI stage IV -28 \\
\cline { 2 - 3 } & CKD stage IV -28 & \\
\hline Grade IV -20 & CKD stage V -44 & MRI stage V-44 \\
\hline \multicolumn{2}{|c|}{ Table-2: Diagram 5: ultrasonographic grading and ADC values } \\
correlation \\
\hline
\end{tabular}

disease was assessed initially. eGFR was calculated as it is the most important indicator of renal function. Various studies have shown accuracy of sonography in assessing the renal parenchymal disease with good sensitivity. ${ }^{17}$ The eGFR is one of the important indicator of reserved renal function and the indicator of prognosis.

In our study we attempted in comparing the sonographic grading of renal parenchymal changes, which is useful in initial evaluation of renal disease with the eGFR which is an indicator of reserved renal function and prognosis. In our study, the correlation between the sonographic grading and the eGFR showed that, increased grading was associated with decreased eGFR. Hence, Sonography can be well used as a preliminary investigating tool, as it is easily available, non-invasive and does not involve exposure to ionizing radiation. By considering eGFR and sonographic grading of renal parenchymal changes the accuracy of assessment of renal ruction will be increased.

In our study, clinically CKD stage I and Stage II, and USG grade I and II cases ADC values are not correlating as most of cases were showing Stage III ADC values. ${ }^{16} \mathrm{ADC}$ values of stage $\mathrm{IV}$ and $\mathrm{V}$ were correlating with $\mathrm{CKD}$ staging and Ultrasound Grading (90 to 100\%) which is similar to Goyal, et $\mathrm{al}^{11}$

The mean $\mathrm{ADC}$ values of renal parenchyma in patients with CKD was significantly lower than in patients with normal renal function, which is similar to Namimoto et al. ${ }^{4}$ reported that $\mathrm{ADC}$ values in both the cortex and the medulla of the kidneys of acute and chronic kidney disease patients were significantly lower than the values in normal population previous investigations. $3,4,5,6,7$

Low ADC values of renal parenchyma explained by reduced perfusion, reduced water diffusion, glomerulosclerosis, tubular atrophy and interstitial fibrosis.Low $\operatorname{ADC}(2.035$ x 10 -3) were seen in renal dysfunction while high $\mathrm{ADC}$ values $(2.451$ $\mathrm{x} 10$-3) were seen in normal function. The mean ADC values in different stages of CKD were different from each othershowing decreasing trend with increasing stages of CKD.

Significant difference was seen with stage $4,5^{15}$ but not with stage $1,2,3$ of CKD. ${ }^{16}$ There was a changing trend observed in incidence of $\mathrm{CKD}$,with renal parenchymal changes observed in younger age groups.

The mean ADC values of different stages of CKD were significantly different from each other and showed decreasing trend with increasing stage $\left(2.01504 \pm 0.1243\left(\times 10^{-3} \mathrm{~mm}^{2} / \mathrm{s}\right)\right.$ for stage-3, $1.8263 \pm 0.2117\left(\times 10^{-3} \mathrm{~mm}^{2} / \mathrm{s}\right)$ for stage- 4 , and $1.2208 \pm 0.1853\left(\times 10^{-3} \mathrm{~mm}^{2} / \mathrm{s}\right)$ for stage -5$)$ which is similar to Goyal, et $\mathrm{al}^{11}$

Within the CKD study group a significant linear correlation was found between renal parenchymal ADC values and stages ofCKD. Similar observations were made by $\mathrm{Xu}$, et al. ${ }^{14}$ who found a linear correlation between renal ADC values and stages of CKD.

There was a significant inverse correlation between ADC values of renal parenchyma and serumCeatininelevels. Similar to our study, Xu, et al. ${ }^{14}$ and Goyal, et al. ${ }^{11}$ found a negative correlation between renal parenchyma ADC values and serum creatinine levels.

\section{Limitations}

We did not recruit healthy volunteers for comparision with renal dysfunction,instead we have taken $\mathrm{ADC}$ values in patients who underwent abdominal MRI for various pathologies with normal renal function

\section{CONCLUSION}

ADC values may serve as additional tool to identify and estimate degree of renal dysfunction as well as to monitor disease progression, $\mathrm{ADC}$ values may help to guide the decision to inject gadolinium based contrast into patients previously known to have renal disease, DWI is not a substitute to serum markers or renal scinitigraphy to asses 
renal dysfunction, rather it is an additional tool, Addition of DWI to existing MRI protocol provide additional imaging information with minimal increase in acquisition time and MRI can be one stop modality for renal evaluation, as it can evaluate Morphology(T1, T2),Pelvicalcyeal system(MR UROGRAPHY), Vascularity (MR ANGIOGRAPHY) and Function (DWI), ADC values of stage IV and $\mathrm{V}$ were correlating with CKD staging and Ultrasound Grading (90 to $100 \%$ ) whereas clinically CKD stage I and Stage II, and USG grade I and II cases ADC values are not correlating as most of cases were showing Stage III ADC values. Linear correlation was seen with ADC values and e GFR and inverse correlation was seen with serum markers, We conclude that $\mathrm{ADC}$ values can play a role in the evaluation of renal dysfunction. Cut off values that we obtained may be useful for stage $3 \mathrm{CKD}$ patients that classified in early stages of disease and respond to treatment.

\section{REFERENCES}

1. Bauer JH, Brooks CS, Burch RN. Clinical appraisal of creatinine clearance as a measurement of glomerular filtration rate. Am J Kidney Dis 1982;2(1):337-46.

2. Prigent A. Monitoring renal function and limitations of renal function tests. SeminNucl Med 2008;38(6):32-46.

3. Yoshikawa T, Kawamitsu H, Mitchell DG, Ohno Y, Ku $\mathrm{Y}$, Seo Y, et al. ADC measurement of abdominal organs and lesions using parallel imaging technique. AJR Am J Roentgenol 2006;187(4):1521-30.

4. Namimoto T, Yamashita Y, Mitsuzaki K, Nakayama Y, Tang Y, Takahashi M. Measurement of the apparent diffusion coefficient in diffuse renal disease by diffusionweighted echo-planar MR imaging. J MagnReson Imaging 1999;9(5):832-37.

5. Thoeny HC, De Keyzer F, Oyen RH, Peeters RR. Diffusion-weighted MR imaging of kidneys in healthy volunteers and patients with parenchymal diseasesinitial experience. Radiology 2005;235(2):911-7.

6. $\mathrm{Xu} \mathrm{Y}$, Wang X, Jiang X. Relationship between the renal apparent diffusion coefficient and glomerular filtration rate: Preliminary experience. J MagnReson Imaging 2007;26(1):678-81.

7. Xu X, Fang W, Ling H, Chai W, Chen K. Diffusionweighted MR imaging of kidneys in patients with chronic kidney disease: Initial study. Eur Radiol 2010;20(3):978-83.

8. Toya R, Naganawa S, Kawai H, Ikeda M. Correlation between estimated glomerular filtration rate (eGFR) and apparent diffusion coefficient (ADC) values of the kidneys. MagnReson Med Sci 2010;9(2):59-64.

9. National Kidney Foundation. K/DOQI clinical practice guidelines for chronic kidney disease: Evaluation, classification, and stratification. Am J Kidney Dis 2002;39(6):S1-266.

10. O'Neill WC. Sonographic evaluation of renal failure. Am J Kidney Dis. 2000;35(6):1021-38.

11. Goyal A, Sharma R, Bhalla AS, Gamanagatti S, Seth A. Diffusion-weighted MRI in assessment of renal dysfunction. Indian J Radiol Imaging [serial online] 2012;22(1):155-9.

12. Cova M, Squillaci E, Stacul F, Manenti G, Gava S,
Simonetti G, et al. Diffusion weighted MRI in the evaluation of renal lesions: Preliminary results. $\mathrm{Br} \mathrm{J}$ Radiol 2004;77(5):851-7.

13. Fukuda Y, Ohashi I, Hanafusa K, Nakagawa T, Ohtani S, Annaka Y, et al. Anisotropic diffusion in kidney: Apparent diffusion coefficient measurements for clinical use. J MagnReson Imaging 2000;11(2):156-60.

14. Xu Y, Wang X, Jiang X. Relationship between the renal apparent diffusion coefficient and glomerular filtration rate: Preliminary experience. J MagnReson Imaging 2007;26(2):678-81.

15. Toya R, Naganawa S, Kawai H, Ikeda M. Correlation between estimated glomerular filtration rate (eGFR) and apparent diffusion coefficient (ADC) values of the kidneys. MagnReson Med Sci 2010;9(1):59-64.

16. Liu H, Zhou Z, Li X, Li C, Wang R, Zhang Y, Niu G. Diffusion-weighted imaging for staging chronic kidney disease: a meta-analysi. Br J Radiol. 2018;91(1091):20170952.

\section{Source of Support: Nil; Conflict of Interest: None}

Submitted: 14-12-2020; Accepted: 16-02-2020; Published online: 14-03-2020 